\title{
Suppression of valid inferences and knowledge structures: The curious effect of producing alternative antecedents on reasoning with causal conditionals
}

\author{
HENRY MARKOVITS and FRÉDÉRIC POTVIN \\ University of Quebec, Montreal, Quebec, Canada
}

\begin{abstract}
These studies looked at the difficulty that reasoners have in accepting conditional ("If P then Q") major premises that are not necessarily true empirically, as a basis for deductive reasoning. Preliminary results have shown that when reasoners are asked to produce possible alternate antecedents to the major premise ("If A then Q"), they paradoxically tend to deny the modus ponens (MP) inference ("If P is true, then Q is true"). Three studies further explored these results. The first study gave university students paper-and-pencil tests in which instructions to "suppose that the major premise is true" was followed by a request to determine the next number in a sequence, to retrieve information unrelated to the premises, or to retrieve a possible case of "If A then Q." Relative to a control group, reasoners asked to produce an alternative antecedent showed a significant tendency to deny the MP inference, whereas no such tendency was observed for the two other tasks used. A second study compared performance on a condition in which reasoners were asked to produce an alternative antecedent with that when they were given an explicit alternative. Premises used in this study were such that the latter alternative antecedent was also spontaneously produced by over $70 \%$ of reasoners. Results showed that the tendency to refuse the MP premise could not be accounted for by the specific nature of the alternative produced. A third study found that the tendency to refuse the MP inference after producing an alternative antecedent was affected by the number of "disabling conditions" (i.e., conditions that allow "P to be true" and "Q to be false") available for the major premise. These results are interpreted as being consistent with a model that supposes that logical reasoning requires selective inhibition of real-world knowledge.
\end{abstract}

The ability to make logical inferences is one of the key components of human reasoning. There has been a great deal of empirical and theoretical attention given to models of how humans make deductions. However, there is one very basic aspect of logical reasoning that has curiously received relatively little attention. One of the critical aspects of logical reasoning is the explicit requirement to disregard the empirical truth of the premises used to make inferences, a requirement that is often expressed by the instructions to "suppose that the premises are true." Yet, although we know that following such instructions is not always easy for even educated reasoners, there is no clear model of just what is involved in doing so, and why this might be difficult. This paper attempts to suggest such a model and to examine it by looking at a paradoxical phenomenon concerning reasoning with causal conditionals.

Preparation of this manuscript was supported by grants from the Fonds pour la Formation de Chercheurs et l'Aide à la Recherche (FCAR) and the Natural Sciences and Engineering Council (NSERC) to H.M. The authors thank David Over and Denise Cummins for their helpful comments on a previous version of this manuscript. Correspondence should be addressed to H. Markovits, Department of Psychology, University of Quebec, C.P. 8888, Succ. “A,” Montreal, PQ, H3C 3P8 Canada (e-mail: markovits.henry@uqam.ca).
Studies of deductive reasoning in both adults and children have clearly shown that people often make deductions that are not consistent with the rules of formal logic. In the context of the focus of this paper, the most interesting of these cases concerns the logical rule known as modus ponens (MP). This requires a reasoner to suppose that a given "If $\mathrm{P}$ then $\mathrm{Q}$ " conditional relation is true, and then to deduce that " $\mathrm{P}$ is true" implies that " $\mathrm{Q}$ is true." Modus ponens is the simplest of the deductive inferences that can be made in conditional logic. Developmental studies have shown that very young children can reliably make the MP inference (Ennis, 1976) before any of the other conditional inferences, such as modus tollens (MT), and so on. Adults generally tend to endorse the MP inference at a very high rate (Evans, 1993). In fact, both of the major theories of human reasoning consider that making the MP inference requires a minimal amount of cognitive effort. Rules theorists (e.g., Braine \& O'Brien, 1991; Rips, 1994) consider MP to be one of the most basic of the inference rules that they claim characterize how people make deductive inferences. Braine (1990) has even proposed that the MP inference rule should be considered a logical primitive with biological origins. Mental model theory considers that the MP inference requires the least costly of the possible representations of the conditional (the "ini- 
tial model"), and should be very easy to make. Both the experimental evidence and theory allow the conclusion that any difficulties in making the MP inference (particularly among adults) cannot be due to the difficulty of actually making the inference once an appropriate representation of the major premise has been established. Thus, examining performance on MP allows focusing on the representational process - that is, what the reasoner must do "to suppose that the major premise is true."

Despite generally high performance on MP, studies have shown that reasoners can be induced to deny the MP inference when prior knowledge about the major premise puts into doubt the empirical truth of the major premise. Several studies have shown that children (Dias \& Harris, 1988; Hawkins, Pea, Glick, \& Scribner, 1984) and young adolescents (Markovits \& Vachon, 1989) tend to deny the MP inference when given premises that are contrary to fact. For example, children who are given a premise such as "If it rains, the street will be dry" will often infer that "the street is wet" when given the minor premise, "it has rained," despite specific instructions to suppose that the major premise is true. Similarly, adults who reason with major premises that they consider to be relatively unbelievable often tend to refuse the MP inference (George, 1995, 1997), in some cases despite explicit instructions to assume the truth of the premises. Other studies have shown that providing additional information that puts into doubt the truth of the major premise can induce adults to deny the MP inference (Byrne, 1989; Chan \& Chua, 1994; Stevenson \& Over, 1995). Byrne (1989), for example, found that while reasoners accepted the MP inference when given the statements "If Mary meets her friend then she will go to the play. Mary meets her friend," they tended to refuse the same inference when the additional premise "If Mary has enough money then she will go to the play" was also presented.

In two other experiments, Cummins and her colleagues (Cummins, 1995; Cummins, Lubart, Alksnis, \& Rist, 1991) measured the number of possible exceptions (referred to as "disabling conditions") to causal conditional rules. These studies showed a clear relationship between performance on MP and the number of possible disabling conditions associated with a given causal relation. MP arguments formed with a premise having many possible disabling conditions, such as "If Joyce eats candy often then she will have cavities. Joyce eats candy often. Therefore, Joyce will have cavities." were judged less certain than those having few possible disabling conditions. Similar results have also been found by Thompson (1994), who examined reasoners' judgments of the relative sufficiency of the major premise.

These results clearly show that there is an interaction between a reasoner's knowledge about the empirical truth of a major premise and their ability to use this premise as a basis for reasoning. When reasoners know that a major premise is not empirically true or when they are given additional information that puts the truth of the premise in doubt, they sometimes find it relatively difficult to "suppose that the premise is true." However, these stud- ies do not really provide any understanding of the kinds of processes that could explain this interaction.

There are probably many differing ways that real-world knowledge can impact reasoning. Certainly some of the previously cited studies have shown that explicitly presented information can have an effect on the type of inferences that reasoners make from any given premises. However, it is also the case that knowledge that is implicitly available also affects reasoning in very clear ways. It is this form of knowledge that interests us in this context.

First, consider reasoning with the two uncertain logical forms that characterize conditional reasoning. Affirmation of the consequent (AC) involves reasoning of the form: "If P then Q. Q is true. Is P true?" Denial of the antecedent (DA) involves reasoning of the form: "If $P$ then Q. P is false. Is Q false?" For both of these inferences, there is no logical conclusion; that is, the correct response is one of uncertainty. The key form of information that affects responding to the two uncertain forms appears to be the existence of possible alternative antecedents to the consequent term of the major premise. For example, for the premise such as "If a rock is thrown at a window, the window will break," possible alternative antecedents could be "throwing a chair at a window," "hitting a window with a bat," and so on. Empirical results have shown a relation between the potential accessibility of alternative antecedents and patterns of reasoning in children (Markovits, Venet, Janveau-Brennan, Malfait, Pion, \& Vadeboncoeur, 1996), the relation between the number of available alternative antecedents and the frequency of correct uncertain conclusions to AC and DA in adolescents (Markovits \& Vachon, 1990) and in adults (Cummins, 1995; Cummins et al., 1991; Thompson, 1994), and the effect of the associative structure of possible antecedents on reasoning in children (Markovits, Fleury, Quinn, \& Venet, 1998) and in adults (Quinn \& Markovits, 1998). These results clearly show that the kinds of inferences that are made to the AC and DA forms is influenced by knowledge that the reasoner has in long-term memory about potential alternative antecedents, although this knowledge is neither explicitly presented nor called for. It is important to also note that in the studies just mentioned, such implicit alternative antecedents have not been found to have any effect on MP.

Performance on MP (and MT) has also been found to be affected by implicit knowledge. For example, Cummins (Cummins, 1995; Cummins et al., 1991) has examined the effects of potential disabling conditions (i.e., conditions that may interact with the antecedent of a given premise to allow the antecedent to be true without the consequent being true) on responding to MP. An example of a disabling condition for the premise "If a rock is thrown at a window the window will break" would be "having a window made of Plexiglas." Cummins has found that reasoners will more often reject the MP (and MT) inference for premises having relatively many disabling conditions. The tendency to refuse the MP inference has also been found to vary with the relative believability of the premise (George, 1995, 1997) or the perceived degree 
of sufficiency of the major premise (Thompson, 1994). Thus, similarly to what has been found for the invalid inferences, accepting the MP inference is clearly influenced by knowledge that the reasoner has in long-term memory about the causal link between antecedent and consequent terms, whether this be stated in terms of disabling conditions or the perceived strength or believability of the causal link between the terms.

How can we understand the way that such implicit information is used by reasoners? Markovits (1993; Markovits et al., 1998; Markovits et al., 1996; Quinn \& Markovits, 1998; Vadeboncoeur \& Markovits, 1999) has proposed that while making a conditional inference, reasoners will automatically activate relevant information in memory concerning the major premise. Information that is activated at a sufficiently strong level will be retrieved and used during reasoning. According to this model, the activation of information related to the conditional proposition is a spontaneous mechanism, which means that people usually have ready access to their knowledge about premises when they reason. The probability of activating relevant information stored in longterm memory is dependent upon the quantity and accessibility of the relevant information.

This model thus assumes that when reasoning with familiar conditionals, reasoners will automatically activate available information about the major premise, including information that might lead them to deny the MP inference. If this information is used for reasoning, then the reasoner will tend to deny the MP inference. What determines whether or not this happens? Vadeboncoeur and Markovits (1999) have recently proposed that the effect of instructions "to suppose that the premises are true" is to allow the reasoner to inhibit the retrieval of information (and the subsequent incorporation into a mental model of the premises) about possible disabling conditions that may have been activated in long-term memory. In support of this position, they found that explicitly activating disabling information modified the effect of the instructions given in the logical task. Specifically, reasoners who were given possible disabling conditions for a major premise found it harder to accept subsequent instructions to disregard this information and to accept the MP premise.

These results are consistent with an inhibition hypothesis. However, they, and others, rely on giving reasoners access to explicit information that clearly affects the way that reasoners interpret the premises that they must reason from. Such forms of information are not part of the normal reasoning process, and it is not clear to what extent these results can be generalized to the more general case of reasoning with only implicitly available information.

The inhibition model supposes that a reasoner will automatically activate the relevant knowledge structure, which includes both information about possible alternative antecedents and that concerning disabling conditions and/or causal sufficiency. Accepting the premises "as true" requires selective inhibition of the latter form of information. This hypothesis allows for the possibility that reactivating the knowledge structure related to the conditional premise subsequent to instructions to "suppose that the major premise is true" will reduce the effects of the previous inhibition, and lead to a greater tendency to deny the MP inference. Clearly, this reactivation cannot be done by explicitly accessing information that suggests that the major premise is not necessarily true, since in this case, there would be no evidence that the reasoner's spontaneous knowledge would ever be accessed. However, suppose that a reasoner can be prompted to reactivate the relevant knowledge structure by explicitly accessing only information that is normally neutral as to the truth of the major premise. In this case, if such a procedure did produce effects on the MP inference, it could be reasonably ascribed to the reactivation of the entire knowledge structure. In fact, results of a previous study (Markovits, Vadeboncoeur, \& Johnson-Laird, 2001) provide one possible way of doing just this. These results provided some preliminary indication that asking reasoners to produce possible alternative antecedents to a given major premise after instructions to "suppose that the premise is true" induces a systematic increase in the tendency to deny the MP inference, one that is not present when antecedents are explicitly presented.

In the following studies, we examine reasoners' responses to MP for causal premises when they are asked to produce alternative antecedents for the major premise.

\section{STUDY 1}

The preliminary results mentioned previously indicate that if the instructions to "suppose that the major premise is true" is followed by instructions to produce one (or more) possible alternatives to the antecedent, then reasoners will tend to deny the MP inference. Now, our basic hypothesis is that this effect is due to reactivation of knowledge about the conditional relation in the major premise. However, there are other possible explanations that must be eliminated before this interpretation can be reasonably made. The first possible explanation for such results is that the simple presence of an intervening task between the instructions and the major premise might be sufficient to confuse reasoners as to what is requested in the primary, logical task - that is, to induce a pragmatic set or simply to induce a set of uncertainty in the reasoner. In order to look at this, we presented reasoners with conditional reasoning problems in one of four conditions. In the neutral condition, we presented the problems with only the standard instructions to suppose that the premise is true. In the calculation condition, we asked reasoners to determine the next number in a mathematical sequence. In the memory condition, we asked reasoners to produce information unrelated to the conditional premise. In the alternatives condition, we asked reasoners to produce an alternative antecedent to the major premise.

\section{Method}

Participants. A total of 114 university students (average age: 25 years 3 months) participated in this study. Of these, 27 received the 
neutral condition, 30 received the calculation condition, 29 received the memory condition, and 28 received the alternatives condition.

Materials. Four versions of a basic test booklet were constructed. Each booklet contained a total of three sets of problems. The front page of each booklet requested basic demographic data (date of birth and sex), followed by these instructions:

"For each of the following three pages, suppose that the sentence at the top of the page is true and reply to the multiple choice questions."

The neutral version contained the following content. On the first page was presented the instruction to "Suppose that it is true that," followed by the major premise "If Julie eats between meals, then she will gain weight." Following this, four questions were asked, corresponding to the four logical forms, MP, DA, AC, and MT. For each question, participants were given a choice of three possible responses. An example of this is the following:

If Julie eats between meals, then one can say:

(a) that it is certain that she will gain weight

(b) that it is certain that she will not gain weight.

(c) that one cannot be certain if she will gain weight or not.

On the second page was presented the instruction to "Suppose that it is true that," followed by the major premise "If Alain sees someone else eating, then he will be hungry." Following this, four questions were asked, corresponding to the four logical forms, AC, MP, MT, and DA. On the third page was presented the instruction to "Suppose that it is true that," followed by the major premise "If Elise exercises, then she will perspire abundantly." Following this, four questions were asked, corresponding to the four logical forms, DA, MT, MP, and AC.

The calculation version was identical to the neutral version except that following each of the three major premises and before the four multiple choice questions, participants were asked to solve mathematical problems involving number sequences. After the premise "If Julie eats between meals, then she will gain weight," the following instructions were given:

Before proceeding, write down the number that would normally be the next one in the following sequence: $1,2,4,8, \ldots$.

After the premise "If Alain sees someone else eating, then he will be hungry," the following instructions were given:

Before proceeding, write down the number that would normally be the next one in the following sequence: $1,4,7,10, \ldots$.

After the premise "If Elise exercises, then she will perspire abundantly," the following instructions were given:

Before proceeding, write down the number that would normally be the next one in the following sequence: $1,3,9,27, \ldots$.

The memory version was identical to the neutral version except that following each of the three major premises and before the four multiple choice questions, participants were asked to retrieve information from memory concerning the person mentioned in the major premise. After the premise "If Julie eats between meals, then she will gain weight," the following instructions were given:

"Julie loves traveling, she has already visited Asia with a group of friends. On the line below, write the name of a country in Asia that she could have visited."

After the premise "If Alain sees someone else eating, then he will be hungry," the following instructions were given:

"Alain is a camping fanatic, so much so that he goes camping all the time. On the line below, write the name of an object that is useful for camping."

After the premise "If Elise exercises, then she will perspire abundantly," the following instructions were given:

"Elise loves anything that concerns the cinema, she knows the names of many actors. On the line below, write the name of an actor that she might know."
The alternatives version was identical to the neutral version except that following each of the three major premises and before the four multiple choice questions, participants were asked to retrieve information from memory concerning the person mentioned in the major premise. After the premise "If Julie eats between meals, then she will gain weight," the following instructions were given:

"Other than eating between meals, there are other reasons that could lead to Julie gaining weight. On the line below, write one of these reasons."

After the premise "If Alain sees someone else eating, then he will be hungry," the following instructions were given:

"Other than seeing someone else eating, there are other reasons that could lead to Alain being hungry. On the line below, write one of these reasons."

After the premise "If Elise exercises, then she will perspire abundantly," the following instructions were given:

"Other than exercising, there are other reasons that could lead to Elise perspiring abundantly. On the line below, write one of these reasons."

Procedure. Questionnaires were distributed at random among entire classes of students. They were given as much time as they needed to respond to the questions.

\section{Results}

The average number of correct responses to each of the four logical forms for the different experimental conditions is given in Table 1. Individual analyses of variance (ANOVAs) for each of the four logical forms were performed with mean number of correct responses as dependent variable and condition as independent variable. For MP, this showed a main effect of condition $[F(3,110)=$ $8.07, p<.001]$. Contrast analysis showed that the number of correct responses to MP was significantly lower in the alternatives condition than in any of the other three. There was no difference in performance between the neutral, calculation, and memory conditions. For MT, this showed a main effect of condition $[F(3,110)=6.46, p<$ $.001]$. Contrast analysis showed that the number of correct responses to MP was significantly lower in the alternatives condition than in any of the other three. There was no difference in performance between the neutral, calculation, and memory conditions. For AC, this showed a main effect of condition $[F(3,110)=3.31, p<.05]$. Contrast analysis showed that the number of correct responses to AC was significantly lower in the memory condition than in the alternatives condition. No other difference was significant. Finally, for DA, the effect of condition was not significant.

Table 1

Percentage of Correct Responses to Each of the Four Logical Forms for the Neutral, Calculation, Memory, and Alternatives Conditions

\begin{tabular}{ccccc}
\hline & \multicolumn{4}{c}{ Condition } \\
\cline { 2 - 5 } Logical Form & Neutral & Calculation & Memory & Alternatives \\
\hline MP & 75.3 & 84.4 & 65.6 & 36.9 \\
MT & 51.9 & 56.7 & 52.9 & 19.0 \\
AC & 72.8 & 70.0 & 56.3 & 85.7 \\
DA & 83.9 & 78.9 & 89.7 & 73.8 \\
\hline
\end{tabular}

MP, modus ponens; MT, modus tollens; AC, affirmation of the consequent; DA, denial of the antecedent. 


\section{Discussion}

A preliminary study showed that when reasoners are asked to produce possible alternative antecedents, they tend to deny the MP inference. The aim of this study was to examine the possibility that the simple existence of an intervening task might either induce a pragmatic set or simply confuse reasoners as to the nature of the primary, logical task. The results are quite clear in this respect. Neither asking participants to make intervening calculations nor asking them to retrieve information unrelated to the major premise has any affect on responding to MP. It is only when reasoners are asked to produce an alternative antecedent that they show a strong tendency to deny the MP inference. The results thus strongly suggest that it is the specific nature of the information retrieved that is responsible for the effect on MP.

\section{STUDY 2}

The results of the first study show that the tendency to accept the MP inference is not affected by the simple presence of an intervening production task. Asking reasoners to produce a possible alternative antecedent does reduce the rate of MP inferences. However, there is still one other possible explanation for these results that must be examined. Previous results (Byrne, 1989; Chan \& Chua, 1994; Stevenson \& Over, 1995) have shown that providing reasoners with explicit alternative antecedents that can be interpreted as preconditions to the consequent of the major premise can affect the rate at which the MP inference is endorsed. This leaves open the general possibility that the specific content of the information that is retrieved by a reasoner who is asked to produce an alternative antecedent might be sufficient to affect responding to MP. The aim of the second study was to look at this possibility.

In order to do this, it was first necessary to find a way to limit the kinds of alternatives that would be spontaneously retrieved in a production task. We did this by providing if-then premises for which there was one very strongly associated cause for the consequent term in the major premise. We then gave reasoners the same task in two basic conditions. In the first, they were asked to produce one alternative to the antecedent of the major premise. In the second, they were explicitly presented with the strongly associated cause as an alternative antecedent. If the rate of acceptance of the MP inference is lower in the production task, then we can conclude that the effect is not due to the specific nature of the retrieved alternative.

\section{Method}

Participants. A total of 110 university students (average age: 22 years 5 months) participated in this study. Of these, 38 received the neutral condition, 36 received the explicit condition, and 36 received the production condition.

Materials. Three versions of a basic test booklet were constructed. Each booklet contained a total of two sets of problems. These problems used two sets of major premises which were each chosen to have a single strongly associated alternative antecedent.
The first two were derived from the results of a previous study (Quinn \& Markovits, 1998):

(1) If a dog has a skin disease, then it will scratch itself constantly. (2) If a dog has soap residues in its skin, then it will scratch itself constantly.

For both of these premises, there is a single strongly associated alternative, which is "having fleas." The second two premises were constructed after an informal pretest:

(3) If a person is psychotic, then they will have hallucinations

(4) If a person has a strong fever, then they will have hallucinations.

For these premises, there is a single strongly associated alternative, which is "taking drugs." Half of the booklets used Premises 1 and 3 in that order, and the other half used Premises 2 and 4 in that order. We used two variants of each basic premise type in order to avoid the possibility of some idiosyncratic interactions between presented and produced antecedents.

The front page of each booklet requested basic demographic data (date of birth and sex), followed by these instructions:

"For each of the following three pages, suppose that the sentence at the top of the page is true and reply to the multiple choice questions."

The neutral version contained the following content. On the first page was presented the instruction to "Suppose that it is true that," followed by the first major premise. Following this, four questions were asked, corresponding to the four logical forms, MP, DA, AC, and MT. For each question, participants were given a choice of two possible responses. An example of this is the following:

If a dog has skin disease, then one can conclude:

(a) that it is certain that it will scratch constantly.

(b) that one cannot be certain if it will scratch constantly or not.

On the second page was presented the instruction to "Suppose that it is true that," followed by the second major premise. Following this, four questions were asked, corresponding to the four logical forms, MP, DA, AC, and MT.

The explicit version was identical to the neutral version except that following each of the two major premises and before the four multiple choice questions, participants were explicitly given the strongly associated alternative. For example:

Suppose that it is true that:

If a dog has a skin disease, then it will scratch itself constantly. It is also true that:

If a dog has fleas, then it will scratch itself constantly.

The production version was identical to the neutral version except that following each of the two major premises and before the four multiple choice questions, participants were asked to generate one possible alternative. For example:

Suppose that it is true that:

If a dog has a skin disease, then it will scratch itself constantly.

On the following line, write one other reason for which a dog would scratch itself constantly:

Procedure. Questionnaires were distributed at random among entire classes of students. They were given as much time as they needed to respond to the questions.

\section{Results}

Table 2 indicates the percentage of correct responses to the four logical forms for each of the three experimental conditions (Table 2). Before looking at these results, we first checked whether in fact the alternatives spontaneously produced by participants were mostly the strongly associated ones. For the premises involving dogs scratching constantly, 25 out of 36 possible responses mentioned 
Table 2

Percent Correct Responses for Each of the Four Logical Forms as a Function of Condition (Neutral, Explicit, Production)

\begin{tabular}{lcccc}
\hline & \multicolumn{4}{c}{ Logical Form } \\
\cline { 2 - 5 } Condition & MP & MT & AC & DA \\
\hline Neutral & $81.6 \%$ & $53.9 \%$ & $84.2 \%$ & $73.7 \%$ \\
Explicit & $69.4 \%$ & $38.9 \%$ & $97.2 \%$ & $84.7 \%$ \\
Production & $44.4 \%$ & $34.7 \%$ & $95.8 \%$ & $90.3 \%$ \\
\hline
\end{tabular}

MP, modus ponens; MT, modus tollens; AC, affirmation of the consequent; DA, denial of the antecedent.

"fleas." For the premises involving hallucinations, 27 out of 36 possible responses mentioned "drugs." This indicated that indeed the premises that were used did spontaneously promote generation of the same alternatives that were presented in the explicit condition. Individual ANOVAs for each of the four logical forms were then performed with mean number of correct responses as dependent variable and condition as independent variable. For MP, this showed a main effect of condition $[F(2,107)=$ $7.52, p<.001]$. Contrast analysis showed that the number of correct responses to MP was significantly lower in the production condition than in any of the other two. There was no difference in performance between the neutral and production conditions. For MT, the effect of condition was not significant. For AC, this showed a main effect of condition $[F(2,107)=3.28, p<.05]$. Contrast analysis showed that the number of correct responses to $\mathrm{AC}$ was significantly lower in the control condition than in either the explicit or the production condition. No other difference was significant. Finally, for DA, the effect of condition was not significant.

Finally, it is possible that although over $70 \%$ of produced alternatives were those used in the explicit condition, the effect might be concentrated in those cases where differing alternatives were in fact generated. In order to examine this possibility, we eliminated all participants in the production condition who did not generate both of the alternatives used in the explicit condition. This resulted in 21 participants in the production condition. Percentage of correct responses of these participants on the four logical forms were as follows: $40.5 \%$ for MP, $40.5 \%$ for MT, $92.9 \%$ for AC, and $90.5 \%$ for DA. As can be seen, the percentage of participants who accepted the MP inference is about the same in this restricted group. Individual ANOVAs showed the same pattern of results for MP as previously found. The only difference in the analyses is that the effect of condition on AC was only marginally significant ( $p=.08$ with the restricted sample).

\section{Discussion}

This study clearly shows that the effect of asking reasoners to produce an alternative antecedent cannot be attributed to the specific nature of the resulting information. In combination with the results of the first study, they provide compelling evidence that the simple production of an alternative antecedent creates a tendency for reasoners to refuse the MP inference that is independent of the nature of the information that is explicitly retrieved.

\section{STUDY 3}

The combined results of the first two studies provide strong evidence for our hypothesis. In the following study, we examined another prediction that can be derived from the inhibition hypothesis. If producing alternative antecedents does have the effect of reactivating the entire knowledge structure concerning the major premise, then the extent to which reasoners subsequently refuse the MP inference should vary as a function of the nature of the information that is activated. Thus, the effect of producing alternative antecedents should not be uniform, but should be constrained by whatever factors influence responding to the MP inference in the absence of specific logical instructions. It has been shown that, in the absence of instructions to "suppose that the major premise is true," reasoners will tend to deny the MP inference to an extent that varies with the potential numbers of disabling conditions (Cummins, 1995; Cummins et al., 1991) and/or the perceived degree of sufficiency of the major premise (Thompson, 1994). We would then predict that the tendency to refuse the MP inference as a result of producing alternative antecedents would be greater for premises for which there is stronger implicit information that puts into doubt the real-world truth of the causal link between the antecedent and consequent.

The specific aim of this study was to examine this prediction. In order to do this in a way that minimized content variation, we constructed pairs of major premises in such a way that the consequent term was identical, but the antecedent was varied in order to allow for more or less potential disabling conditions. For example, the following premise was chosen to have relatively few disabling conditions:

If Jean has a severe accident, then he will be late for his meeting.

The next premise was chosen to have relatively many disabling conditions, while maintaining the same consequent.

If there is a snow storm, then Jean will be late for his meeting.

Thus, participants were given premises with few or many disabling conditions in either the neutral condition or the alternatives conditions, previously described. We predicted that requesting production of an alternative antecedent would significantly decrease acceptance of the MP inference for these premises relative to the neutral presentation, but that this decrease would be greater for premises with many disabling conditions than for those with few disabling conditions.

\section{Method}

Participants. A total of 359 university students (average age: 23 years 2 months) participated in this study. Of these, 92 received premises with few disabling conditions in the neutral condition, 90 received premises with many disabling conditions in the neutral condition, 87 received premises with few disabling conditions in the alternatives condition, and 28 received premises with many disabling conditions in the alternatives condition. 
Materials. Eight versions of a basic test booklet were constructed. Each booklet contained a total of three sets of problems. The basic format of the booklets was identical to that described in the first study. Half of the booklets presented the reasoning problems in the neutral condition, while in the other half, problems were presented in the alternatives condition.

In order to construct the premises for the reasoning problems, we used six basic consequents. This gave six premises with few disabling conditions and six others with many disabling conditions. The following are the premises that were considered to have relatively few disabling conditions:

(1) If Jean has a severe accident, then he will be late for his meeting.

(2) If a window pane is violently struck with an steel bar, then the window pane will break.

(3) If there is an electric storm, then there will be a noise.

(4) If Julie is pregnant, then she will gain weight.

(5) If Alain is deprived of food for a long time, then he will be hungry.

(6) If Elise is in a sauna that is working at its maximum, then she will perspire abundantly.

The following premises were considered to have relatively many disabling conditions:

(7) If there is a snow storm, then Jean will be late for his meeting.

(8) If a window pane is struck with a stone, then the window pane will break.

(9) If a plate is dropped, then there will be a noise.

(10) If Julie eats between meals, then she will gain weight.

(11) If Alain sees someone else eating, then he will be hungry.

(12) If Elise exercises, then she will perspire abundantly.

In order to minimize the number of problems that a single participant was asked to resolve, all test booklets contained only three problem sets. Two booklets with premises having few disabling conditions in the neutral and the alternatives conditions, respectively, were constructed using Premises 1-3. Two other booklets with premises having few disabling conditions in the neutral and the alternatives conditions, respectively, were constructed using Premises 4-6. Two booklets with premises having many disabling conditions in the neutral and the alternatives conditions, respectively, were constructed using Premises 7-9. Two other booklets with premises having many disabling conditions in the neutral and the alternatives conditions, respectively, were constructed using Premises 10-12.

Procedure. Questionnaires were distributed at random among entire classes of students. They were given as much time as they needed to respond to the questions.

\section{Results}

We first performed a manipulation check in order to verify whether the premises that we used did indeed differ as presumed. We first asked 23 students, who did not take part in this experiment, to indicate for half of the premises used as many potential disabling conditions as they could easily think of. Each of the participants received half of the premises with few and half with many disabling. For the premises with few disabling conditions, the mean number thus produced was 0.87 ; for the premises with many disabling conditions, the mean was 1.47. A paired $t$ test showed that there were significantly more disabling conditions produced on the premises with many than with few disabling conditions. We also asked 21 students to rate each of the premises as to the strength of the causal link between antecedent and consequent, on a scale from 1 to 7 . Mean ratings were 4.97 for premises with few disabling conditions and 3.44 for those with many disabling conditions.

We then examined responses to the logical questions. Table 3 indicates the mean number of correct responses for each of the four logical forms as a function of the relative number of disabling conditions (few, many) and whether or not reasoners were asked to produce a possible alternative antecedent (Table 3). Individual ANOVAs were performed for each of the four logical forms, with mean number of correct responses as dependent variable and type of premise (few, many) and condition (production of alternatives, none) as independent variables. For MP, significant main effects of type of premise $[F(1,355)=$ $13.87, p<.001]$ and condition $[F(1,355)=31.87, p<$ $.001]$, as well as a significant type of premise $\times$ condition interaction $[F(1,355)=6.48, p<.01]$, were found. Contrast analyses showed that fewer participants accepted the MP inference when asked to produce an alternative $(66.1 \%)$ than when not asked to do so $(87.0 \%)$. In addition, when reasoners produced an alternative antecedent, the MP inference was accepted more often for premises with few possible disabling conditions $(77.8 \%)$ than for premises with many possible disabling conditions $(54.8 \%)$. When reasoners did not produce an alternative antecedent, there was no significant difference between the two types of premise. For MT, the ANOVA indicated significant main effects of type of premise $[F(1,355)=4.23, p<.05]$ and condition $[F(1,355)=$ $14.86, p<.001]$. Contrast analyses showed that fewer participants accepted the MT inference when asked to produce an alternative $(46.5 \%)$ than when not asked to do so $(62.6 \%)$. In addition, fewer participants accepted the MT inference for premises with many possible disabling conditions $(50.4 \%)$ than for premises with few disabling con-

Table 3

Percent Correct Responses for Each of the Four Logical Forms as a Function of Premise Type (Few Disabling Conditions, Many Disabling Conditions) and Experimental Condition (Production of Alternative Antecedents, None)

\begin{tabular}{ccccc}
\hline & \multicolumn{4}{c}{ Premise Type } \\
\cline { 2 - 5 } $\begin{array}{c}\text { Logical } \\
\text { Form }\end{array}$ & $\begin{array}{c}\text { None (Few) } \\
(n=92)\end{array}$ & $\begin{array}{c}\text { None (Many) } \\
(n=90)\end{array}$ & $\begin{array}{c}\text { Production (Few) } \\
(n=87)\end{array}$ & $\begin{array}{c}\text { Production (Many) } \\
(n=90)\end{array}$ \\
\hline MP & 89.1 & 84.8 & 77.8 & 54.8 \\
MT & 63.8 & 61.5 & 54.0 & 39.3 \\
AC & 82.2 & 75.2 & 95.4 & 89.3 \\
DA & 85.1 & 71.9 & 92.0 & 91.1 \\
\hline
\end{tabular}

MP, modus ponens; MT, modus tollens; AC, affirmation of the consequent; DA, denial of the antecedent. 
ditions (59.0\%). For AC, the ANOVA indicated significant main effects of type of premise $[F(1,355)=4.96$, $p<.05]$ and condition $[F(1,355)=21.08, p<.001]$. Contrast analyses showed that more participants correctly refused the $\mathrm{AC}$ inference when asked to produce an alternative $(92.3 \%)$ than when not asked to do so (78.7\%). In addition, more participants refused the AC inference for premises with few possible disabling conditions $(88.8 \%)$ than for premises with many disabling conditions $(82.2 \%)$. For DA, the ANOVA indicated significant main effects of type of premise $[F(1,355)=6.39$, $p<.01]$ and condition $[F(1,355)=21.73, p<.001]$, as well as a significant type of premise $\times$ conditions interaction $[F(1,355)=4.96, p<.05]$. Contrast analyses showed that more participants correctly refused the DA inference when asked to produce an alternative $(91.5 \%)$ than when not asked to do so $(78.5 \%)$. In addition, more participants refused the DA inference for premises with few possible disabling conditions $(85.1 \%)$ than for premises with many disabling conditions (71.9\%), but only when not asked to produce an alternative antecedent.

\section{Discussion}

There are basically two components to these results. The first concerns the predictable effect of producing alternative antecedents on responding to the two uncertain forms, AC and DA. Having increased access to alternative antecedents has been frequently shown to increase the proportion of uncertainty responses to both these forms, and the present results are consistent with this effect. The critical part of the results is the effect of producing alternative antecedents on responding to MP. First, it is important to note that when participants were given instructions to "suppose that it is true that," the rate of acceptance of the MP inference was uniformly high, irrespective of the nature of the premises. Following this instruction by a request to produce an alternative antecedent results in an increased tendency to refuse this inference. Critically, and as predicted, the resulting tendency to deny MP varies according to the relative number of potential disabling conditions. Following production of alternative antecedents, reasoners deny the MP inference more often when given premises for which there are relatively more disabling conditions (and for which the causal link between antecedent and consequent is weaker).

\section{GENERAL DISCUSSION}

These three studies provide some clear support for the existence of a seemingly paradoxical effect. They show that when a reasoner is asked "to suppose that (an if-then major premise) is true" and then asked to produce an alternative antecedent to the consequent of this premise, they will tend to refuse the MP inference. Our results show that the effect of producing an alternative on the tendency to accept the MP inference is clearly greater than the potential effects of the general nature of the production task or the specific nature of the information that is generated by reasoners. This basic result must be un- derstood in a larger context. First, the present results show that educated adults have little difficulty in accepting the premises used in this study "as true," even though they clearly possess knowledge suggesting that the causal relation in the major premise is in fact not necessarily true. Second, previous studies have shown that although the amount of information that reasoners have about potential alternatives to the antecedent is related to how they reason with the AC and DA logical forms, it has no effect on reasoning with MP (Cummins, 1995; Cummins et al., 1991; Markovits \& Vachon, 1990; Thompson, 1994).

Now, a key factor in understanding what these results mean is the specific difference between producing and presenting a given alternative. The first study shows that the tendency to refuse the MP inference is clearly present only when an alternative antecedent is produced. However, the second study also shows that the effect of producing a given alternative antecedent is much stronger than that obtained by explicitly presenting this same alternative to a reasoner. Thus, it is highly unlikely that this effect can be explained solely by retrieval of the specific alternative from memory. However, it is also the case that when information is activated in memory, other, semantically related information is also activated. Thus, a compelling conclusion from these results is that the tendency to denial of the MP inference in the production condition is produced by the activation of associated information that puts the truth of the major premise in doubt, that is, potential disabling conditions and/or judgments of sufficiency. This conclusion is clearly reinforced by the results of the third study, which show that asking reasoners to produce an alternate antecedent after presentation of the major premise produces a tendency to deny the MP inference that is related to the quantity of implicitly available information that puts in doubt the sufficiency of the if-then premise. Our results are thus consistent with our basic hypothesis - that is, that production of an alternative antecedent also activates associated information (such as disabling conditions) that put in doubt the strength of the if-then relation presented in the major premise.

What, then, does this imply as to the nature of "logical" reasoning. We must first introduce an important caveat here. The results of these studies, and of many others, do show that a significant proportion of even educated adults appear to reason with meaningful premises in ways that are consistent with the model that we have presentedthat is, even when making "logical" inferences, they will activate and use their knowledge of the premises. This does not imply that more expert reasoners are not capable of more abstract strategies (Venet \& Markovits, 2001). However, developmental studies, previous studies with university students, and the present results indicate that expert reasoners are relatively rare and relatively old. But for what appears to be a strong majority of reasoners, the first implication of these results is that reasoning that "supposes that the premises are true," irrespective of their empirical status, is not really distinct from ordinary real-world reasoning. The present studies imply that, irrespective of the nature of the instructions used to determine the kind 
of inference required, reasoners retain access to their knowledge of the specific "if-then" relation that is in the major premise. In any case, some of the resulting information is necessary in making appropriate inferences, whether or not these are "logical." For example, accessing potential alternative antecedents makes it less likely for a reasoner to make the incorrect inference that " $\mathrm{P}$ implies Q. Q is true then P must be true." Although this inference is logically invalid, it is also pragmatically incorrect if there are in fact alternative antecedents of the form "A implies Q." Thus, what appears to be a critical factor in determining the "kind" of inferences that are made by most reasoners is the choice of information that is used. Instructions to "suppose that the premises are true" are used to attempt to selectively inhibit retrieval of information that would put into doubt the truth of these premises. And, as we have seen, this process of selective inhibition is quite fragile.

The analysis that we have made of reasoning is also consistent with the notion that inhibition may be a key factor in understanding cognitive performance. This is particularly true in a developmental perspective. Several authors have focused on inhibition as a key factor in the cognitive difficulties of younger children (e.g., Bjorklund \& Harnishfeger, 1990). Consistent with this, recent developmental studies concerning conditional reasoning (Janveau-Brennan \& Markovits, 1999; Markovits et al., 1998) have shown that in primary school children, increased access to potential disabling conditions for if-then premises with content that was familiar to them is in fact paralleled by an increase in the tendency to refuse the MP inference.

\section{REFERENCES}

BJorklund, D. F., \& HARnishfeger, K. K. (1990). The resources construct in cognitive development: Diverse sources of evidence and a theory of inefficient inhibition. Developmental Review, 10, 48-71.

Braine, M. D. S. (1990). The "natural logic" approach to reasoning. In W. F. Overton (Ed.), Reasoning, necessity and logic: Developmental perspectives (pp. 133-157). Hillsdale, NJ: Erlbaum.

Braine, M. D. S., \& O’Brien, D. P. (1991). A theory of if: Lexical entry, reasoning program, and pragmatic principles. Psychological Review, 98, 182-203.

By RNE, R. M. J. (1989). Suppressing valid inferences with conditionals. Cognition, 30, 61-83.

Chan, D., \& ChuA, F. (1994). Suppression of valid inferences: Syntactic views, mental models, and relative salience. Cognition, 53, 217-238.
Cummins, D. D. (1995). Naive theories and causal deduction. Memory \& Cognition, 23, 646-658.

Cummins, D. D., Lubart, T., Alksnis, O., \& Rist, R. (1991). Conditional reasoning and causation. Memory \& Cognition, 19, 274-282.

DiAs, M. G., \& HARRIS, P. L. (1988). The effect of make-believe play on deductive reasoning. British Journal of Developmental Psychology, 6, 207-221.

ENNIS, R. H. (1976). An alternative to Piaget's conceptualization of logical competence. Child Development, 47, 903-919.

Evans, J. ST. B. T. (1993). The mental model theory of conditional reasoning: Critical appraisal and revision. Cognition, 48, 1-20.

GEORGE, C. (1995). The endorsement of the premises: Assumption-based or belief-based reasoning. British Journal of Psychology, 86, 93-111.

GEORGE, C. (1997). Reasoning from uncertain premises. Thinking \& Reasoning, 3, 161-189.

Hawkins, J., Pea, R. D., Glick, J., \& Scribner, S. (1984). "Merds that laugh don't like mushrooms": Evidence for deductive reasoning by preschoolers. Developmental Psychology, 20, 584-594.

JanVEAU-Brennan, G., \& Markovits, H. (1999). The development of reasoning with causal conditionals. Developmental Psychology, 35, 904-911.

Markovits, H. (1993). The development of conditional reasoning: A Piagetian reformulation of the theory of mental models. Merrill-Palmer Quarterly, 39, 133-160.

Markovits, H., Fleury, M.-L., Quinn, S., \& Venet, M. (1998). Conditional reasoning and the structure of semantic memory. Child Development, 64, 742-755.

MARKovits, H., \& VACHON, R. (1989). Reasoning with contrary-to-fact propositions. Journal of Experimental Child Psychology, 47, 398-412.

Markovits, H., \& VACHON, R. (1990). Conditional reasoning, representation and level of abstraction. Developmental Psychology, 26, 942-951.

Markovits, H., Vadeboncoeur, I., \& Johnson-Laird, P. N. (2001). On the interpretation of conditionals in deductive reasoning. Unpublished manuscript.

Markovits, H., Venet, M., Janveau-Brennan, G., Malfait, N., Pion, N., \& VAdEBoncoeUr, I. (1996). Reasoning in young children: Fantasy and information retrieval. Child Development, 67, 2857-2872.

Quinn, S., \& MARKovits, H. (1998). Conditional reasoning, causality, and the structure of semantic memory: Strength of association as a predictive factor for content effects. Cognition, 68, B93-B101.

RIPS, L. J. (1994). The psychology of proof: Deductive reasoning in human thinking. Cambridge, MA: MIT Press.

Stevenson, R. J., \& Over, D. E. (1995). Deduction from uncertain premises. Quarterly Journal of Experimental Psychology, 48A, 613-643.

Thompson, V. A. (1994). Interpretational factors in conditional reasoning. Memory \& Cognition, 22, 742-758.

VAdeboncoeur,I., \& Markovits, H. (1999). The effect of instructions and information retrieval on accepting the premises in a conditional reasoning task. Thinking \& Reasoning, 5, 97-113.

VENET, M., \& MARKovits, H. (2001). Understanding uncertainty with abstract conditional premises. Merrill-Palmer Quarterly, 47, 74-99.

(Manuscript received February 16, 2000; revision accepted for publication February 19, 2001.) 